\title{
- COMUNICAÇÃO CIENTÍFICA - \\ CANIBALISMO DE OVOS POR FÊMEAS DE CHRYSOPERLA EXTERNA (HAGEN) (NEUROPTERA, CHRYSOPIDAE) EM CRIAÇÕES DE LABORATÓRIO
}

\begin{abstract}
Alexandre Pinho de Moura - Departamento de Entomologia e Fitopatologia, Instituto de Biologia, Universidade Federal Rural do Rio de Janeiro (UFRRJ), apmoura@ufrrj.br

Débora Candeias Marques de Moura - Departamento de Engenharia, Universidade Federal de Lavras (UFLA), debcandeias@yahoo.com.br
\end{abstract}

\section{RESUMO}

Dentre as diversas espécies de Chrysopidae criadas massalmente e utilizadas em programas de manejo como controladores biológicos de pragas agrícolas, aquelas pertencentes ao gênero Chrysoperla Steinmann merecem destaque. Este trabalho avaliou a ocorrência de canibalismo de ovos por adultos de Chrysoperla externa (Hagen) (Neuroptera, Chrysopidae) em criações de laboratório. Adultos dessa espécie de crisopídeo foram criados em gaiolas de PVC (20 cm de diâmetro por $20 \mathrm{~cm}$ de altura), na densidade de 60 casais por unidade de criação. Aos adultos foi fornecida água destilada, sendo os mesmos alimentados com dieta artificial à base de levedo de cerveja e mel, na proporção de 1:1 (v:v). Em todas as unidades de criação ocorreu canibalismo de ovos por fêmeas de C. externa, sendo que algumas dessas fêmeas predavam seus próprios ovos recém-depositados. Não foi constatado canibalismo por machos dessa espécie.

Palavras-chave: controle biológico; crisopídeo; nutrição.

\section{ABSTRACT}

Among the several species of Chrysopidae mass reared and utilized in management programs as biological controllers of agricultural pests, those belonging to the genus Chrysoperla Steinmann deserve attention. This work evaluated the egg cannibalism occurrence by adults of Chrysoperla externa (Hagen) (Neuroptera, Chrysopidae) in laboratory rearing. Adults of that green lacewing species were reared in cylindrical cage $(20 \mathrm{~cm}$ diameter x $20 \mathrm{~cm}$ height), with 60 couples by cage. Adults were fed with artificial diet based on brewer's yeast and honey 1:1 (v:v). Distilled water was provided to the insects. In all rearing cages the cannibalism of eggs by females of $C$. externa was observed, being that some females fed on their own newly-laid eggs. It was not verified cannibalism by males of that green lacewing species.

Key-words: biological control; green lacewing; nutrition.

Insetos pertencentes à família Chrysopidae têm sido relatados como predadores que apresentam elevado potencial biótico, alta voracidade e capacidade de adaptação a diferentes agroecossistemas. Atuam como predadores alimentando-se de pulgões, cochonilhas, moscas-brancas, ovos e lagartas neonatas de lepidópteros, tripes e ácaros, além de muitos outros artrópodes de pequeno tamanho e tegumento facilmente perfurável (Carvalho \& Souza, 2002; Medina et al., 2003; Athan et al., 2004).

A família Chrysopidae é representada por cerca de 86 gêneros e mais de 1.200 espécies, apresentando ampla distribuição geográfica, sendo que muitas das espécies são encontradas na região Neotropical (Brooks \& Barnard, 1990). No Brasil, 81 espécies já foram descritas, estando distribuídas em seis gêneros e dois subgêneros (Freitas \& Penny, 2001), dos quais o gênero Chrysoperla Steinmann, 
1964 é o mais estudado. Os insetos desse gênero são os mais utilizados em programas de manejo como controladores biológicos de pragas agrícolas (Tauber et al., 2000; Freitas \& Penny, 2001).

Muitas espécies de crisopídeos são predadoras somente na fase larval, sendo a alimentação dos adultos constituída de pólen, 'honeydew' (excreção de afídeos e de algumas cochonilhas) e exsudatos de plantas. Entretanto, em algumas espécies, a fase adulta também atua como predadora (Carvalho \& Souza, 2000).

Assim sendo, objetivou-se com o presente trabalho avaliar a ocorrência de canibalismo por adultos de Chrysoperla externa (Hagen) (Neuroptera, Chrysopidae), em criações de laboratório.

O estudo foi realizado no Laboratório de Biologia de Insetos do Departamento de Entomologia da Universidade Federal de Lavras (UFLA), em Lavras, Minas Gerais, utilizandose de uma população de $C$. externa coletada em pomares comerciais de macieira no município de Vacaria, Rio Grande do Sul.

Adultos dessa espécie de crisopídeo foram criados em gaiolas de cloreto de polivinila (PVC) de $20 \mathrm{~cm}$ de diâmetro e $20 \mathrm{~cm}$ de altura, revestidas internamente com papel-filtro branco, na densidade de 60 casais por unidade de criação (um casal por $105 \mathrm{~cm}^{3}$ ), próxima à densidade de um casal por $110 \mathrm{~cm}^{3}$, conforme sugerido por Carvalho (1994) e Carvalho \& Souza (2000), utilizando-se de oito gaiolas. As gaiolas foram apoiadas sobre bandejas plásticas de $25 \mathrm{~cm}$ de diâmetro, forradas com o mesmo tipo de papel, sendo a parte superior de cada gaiola fechada com filme de PVC. As gaiolas foram mantidas em sala climatizada a $25 \pm 2^{\circ} \mathrm{C}$, UR de $70 \pm 10 \%$ e fotofase de 12 horas.

Os insetos foram alimentados com dieta artificial à base de levedo de cerveja e mel na proporção volumétrica de 1:1 (Barbosa et al., 2002), considerada adequada para a fase adulta (Carvalho \& Souza, 2000), que foi pincelada em duas tiras de Parafilm ${ }^{\circledR}$ de $10 \mathrm{~cm}$ de comprimento por $2 \mathrm{~cm}$ de largura, fixadas na parede interna de cada uma das gaiolas. No interior de cada gaiola de criação foi colocado um recipiente de vidro com capacidade para $10 \mathrm{~mL}$ contendo algodão hidrófilo saturado com água destilada, de modo a garantir o fornecimento de água aos insetos, atuando, também, como umidificador (Carvalho \& Souza, 2000). Avaliou-se a ocorrência de canibalismo nas gaiolas de criação por um período de uma hora de observação para cada gaiola.

Verificou-se que em todas as unidades de criação ocorreu canibalismo de ovos por fêmeas de $C$. externa, sendo que algumas dessas fêmeas predavam seus próprios ovos recém-depositados, ou mesmo ovipositava-os diretamente em seu aparelho bucal, curvando o abdômen nessa direção. No entanto, não foi observado canibalismo por machos, o que sugere que esse comportamento, exclusivo de fêmeas, seja devido, provavelmente, ao seu maior requerimento nutricional quando em comparação aos machos.

Acredita-se, ainda, que esses insetos, quando criados em laboratório, podem estar expostos a situações de estresse freqüente, devido às manipulações das unidades de criação, o que pode interferir sobremaneira na quantidade de alimento ingerido, quer seja pelas larvas ou mesmo pelos adultos, culminando com a obtenção de adultos portadores de distúrbios fisiológicos e/ou comportamentais. Segundo Carvalho \& Souza (2000), a quantidade e a qualidade da dieta ingerida pelas larvas de crisopídeos afeta não somente o desenvolvimento dessa fase, mas também das fases subseqüentes. Uma deficiência trófica durante a vida da larva, não pode ser compensada pela utilização de uma dieta considerada adequada para o imago.

Apesar de adultos das espécies de Chrysoperla serem referenciados alimentandose exclusivamente de substâncias açucaradas e de pólen (Principi \& Canard, 1984), acredita- 
se, diante dos relatos apresentados no presente estudo, que a espécie $C$. externa possa atuar como predadora de artrópodes-praga também durante a fase adulta, o que já foi relatado por outros autores (Ru et al., 1975; Núñez, 1985; Núñez, 1988). Tauber \& Tauber (1973), por sua vez, já haviam relatado que adultos de Chrysoperla carnea (Stephens) (Neuroptera, Chrysopidae) "strain Mohave" são capazes de predar afídeos. No entanto, pesquisas visando à confirmação da potencialidade de utilização de adultos de $C$. externa como predadores de artrópodes-praga em agroecossistemas necessitam ser realizadas.

Diante dos relatos apresentados, conclui-se que: 1) ocorre canibalismo de ovos por fêmeas adultas de $C$. externa em criações de laboratório; 2) o canibalismo de ovos por adultos dessa espécie de crisopídeo não é observado para machos; 3) novas pesquisas necessitam ser desenvolvidas, com o intuito de confirmar a possibilidade da utilização de adultos de $C$. externa como predadores de artrópodes-praga.

\section{AGRADECIMENTOS}

Ao Conselho Nacional de Desenvolvimento Científico e Tecnológico - CNPq, pela concessão de bolsa de doutorado ao primeiro autor.

\section{REFERÊNCIAS BIBLIOGRÁFICAS}

ATHAN, R.; KAYDAN, B.; OZGOKCE, M.S. Feeding activity and life history characteristics of the generalist predator, Chrysoperla externa (Neuroptera: Chrysopidae) at different prey densities. Journal of Pest Science, New York, v.77, n.1, p.17-21, 2004.

BARBOSA, L.R.; FREITAS, S.; AUAD, A.M. Capacidade reprodutiva e viabilidade de ovos de Ceraeochrysa everes (Banks, 1920) (Neuroptera: Chrysopidae) em diferentes condições de acasalamento. Ciência e Agrotecnologia,
Lavras, v.26, n.3, p.466-471, 2002.

BROOKS, S.J.; BARNARD, P.C. The green lacewings of the world: genus Chrysoperla (Neuroptera: Chrysopidae). Bulletin of the British Museum Natural History (Entomology), London, v.63, n.2, p.117-286, 1990.

CARVALHO, C.F. Analyse des éléments du potentiel reproducteur em vue de la production de Chrysoperla mediterranea (Hölzel, 1972) (Neuroptera: Chrysopidae). Toulouse, França, Université Paul-Sabaatier, 1994. 164p. (Tese de Doutorado).

CARVALHO, C.F.; SOUZA, B. Potencial de insetos predadores no controle biológico aplicado. In: PARRA, J.R.P.; BOTELHO, P.S.M.; CORRÊA-FERREIRA, B.S.; BENTO, J.M.S. (Eds.). Controle biológico no Brasil: parasitóides e predadores. São Paulo: Manole, 2002. p.191-208.

CARVALHO, C.F.; SOUZA, B. Métodos de criação e produção de crisopídeos. In: BUENO, V.H.P. (Ed.). Controle biológico de pragas: produção massal e controle de qualidade. Lavras: UFLA, 2000. p.91-109.

FREITAS, S.; PENNY, N.D. The green lacewings (Neuroptera: Chrysopidae) of brazilian agroecosystems. Proceedings of the California Academy of Science, San Francisco, v.52, n.19, p.245-395, 2001.

MEDINA, P.; SMAGGHE, G.; BUDIA, F.; TIRRY, L.; VIÑUELA, E. Toxicity and absorption of azadirachtin, diflubenzuron, pyriproxyfen and tebufenozide after direct spray in predatory larvae of Chrysoperla carnea (Stephens) (Neuroptera: Chrysopidae). Environmental Entomology, Lanham, v.32, n.1, p.196-203, 2003. 
NÚÑEZ, S.E. Primer registro y utilización en el Perú de Chrysoperla externa (Hagen) y Ceraeochrysa cincta Schneider. Informativo del Área Andina, Lima, v.4, n.1, p.1-19, 1985.

NÚÑEZ, E. Ciclo biológico y crianza de Chrysoperla externa y Ceraeochrysa cincta (Neuroptera, Chrysopidae). Revista Peruana de Entomología, Lima, v.31, n.1, p.76-82, 1988.

PRINCIPI, M.M.; CANARD, M. Feeding habits. In: CANARD, M.; SÉMÉRIA, Y.; NEW, T.R. (Eds.). Biology of Chrysopidae. The Hague: Dr. W. Junk Publishers, Boston, 1984. p.76-92.

RU, N.; WHITCOMB, W.H.; MURPHEY, M.;
CARLYSLE, T.C. Biology of Chrysopa lanata (Neuroptera: Chrysopidae). Annals of the Entomological Society of America, Lanham, v.68, n.2, p.187-190, 1975.

TAUBER, M.J.; TAUBER, C.A.; DANNE, K.M.; HAGEN, K.S. Commercialization of predators: recent lessons from the green lacewings (Neuroptera: Chrysopidae: Chrysoperla). American Entomologist, Lanham, v.46, n.1, p.26-38, 2000.

TAUBER, M.J.; TAUBER, C.A. Nutritional and photoperiodic control of the seasonal reproductive cycle in Chrysopa mohave (Neuroptera, Chrysopidae). Journal of Insect Physiology, Oxford, v.19, n.4, p.729-736, 1973. 